\title{
Glycaemic control in the paediatric and young adult population with type 1 diabetes following a single telehealth visit - what have we learned from the COVID-19 lockdown?
}

\author{
Marianna Rachmiel ${ }^{1,2}$ (1) $\cdot$ Yael Lebenthal ${ }^{2,3}$ (1) $\cdot$ Kineret Mazor-Aronovitch ${ }^{2,4,5} \cdot$ Avivit Brener $^{2,3}$ (1) $\cdot$ Noa Levek $^{4,5}$. \\ Neria Levran ${ }^{4,5} \cdot$ Efrat Chorna $^{3} \cdot$ Michal Dekel $^{1}$. Galia Barash ${ }^{1,2} \cdot$ Zohar Landau $^{4,6,7}$ (1) - Orit Pinhas-Hamiel ${ }^{2,4,5}$ (i)
}

Received: 23 August 2020 / Accepted: 7 January 2021 / Published online: 28 January 2021

@ Springer-Verlag Italia S.r.l., part of Springer Nature 2021

\begin{abstract}
Aims Children with chronic diseases were unable to receive their usual care during COVID-19 lockdown. We assessed the feasibility and impact of telehealth visits on the time-in-range (TIR) of paediatric individuals with type 1 diabetes (T1D). Methods An observational multicentre real-life study. Patients scheduled for an in-clinic visit during the lockdown were offered to participate in a telehealth visit. Sociodemographic, clinical, continuous glucose monitor and pump data were recorded 2 weeks prior and 2 weeks after telehealth visit. The primary endpoint was change in relative-TIR, i.e. change in TIR divided by the percent of possible change ( $\Delta$ TIR/(100-TIRbefore)*100).

Results The study group comprised 195 individuals with T1D (47.7\% males), mean \pm SD age $14.6 \pm 5.3$ years, and diabetes duration $6.0 \pm 4.6$ years. Telehealth was accomplished with 121 patients and their parents (62.0\%); 74 (38.0\%) did not transfer complete data. Mean TIR was significantly higher for the two-week period after the telehealth visit than for the two-week period prior the visit $(62.9 \pm 16.0, p<0.001$ vs. $59.0 \pm 17.2)$; the improvement in relative-TIR was $5.7 \pm 26.1 \%$. Initial higher mean glucose level, lower TIR, less time spent at $<54 \mathrm{mg} / \mathrm{dl}$ range, longer time spent at $180-250 \mathrm{mg} / \mathrm{dl}$ range, higher daily insulin dose, and single-parent household were associated with improved relative-TIR. Multiple regression logistic analysis demonstrated only initial lower TIR and single-parent household were significant, odds ratio: $-0.506,(95 \% \mathrm{CI}-0.99,-0.023)$, $p=0.04$ and 13.82, (95\%CI 0.621, 27.016), $p=0.04$, respectively.

Conclusions Paediatric and young adult patients with T1D benefited from a telehealth visit during COVID-19. However, this modality is not yet suitable for a considerable proportion of patients.
\end{abstract}

Keywords Children · Adolescents · CGM metrics · Time-in-range $\cdot$ Telehealth $\cdot$ Telemedicine

$\begin{array}{ll}\text { Abbreviations } \\ \text { ADHD } & \text { Attention-deficit hyperactivity disorder } \\ \text { CGM } & \text { Continuous glucose monitoring } \\ \text { GMI } & \text { Glycaemic metabolic index } \\ \text { TIR } & \text { Time in range }\end{array}$

Managed by Massimo Federici

Marianna Rachmiel

mariannar@shamir.gov.il

1 Pediatric Endocrinology Unit, Shamir (Assaf Harofeh) Medical Center, Tzrifin, Israel

2 Sackler Faculty of Medicine, Tel Aviv University, Tel Aviv, Israel

3 Pediatric Endocrinology and Diabetes Unit, Dana-Dwek Children's Hospital, Tel Aviv Sourasky Medical Center, Tel Aviv, Israel

\section{Introduction}

According to standards of medical care for individuals with type 1 diabetes (T1D), interval follow-up visits should occur at least every 3-4 months, individualized to the patient $[1,2]$. These visits are for patients' education, empowerment, and

4 National Juvenile Diabetes Center, Maccabi Health Care Services, Ra'anana, Israel

5 Pediatric Endocrine and Diabetes Unit, Chaim Sheba Medical Center, Edmond and Lily Safra Children's Hospital, Ramat-Gan, Israel

6 Pediatrics Department, Barzilai Medical Center, Ashkelon, Israel

7 Faculty of Health Sciences, Ben-Gurion University of the Negev, Beer-Sheva, Israel 
review of care plans. We presume that its rationale is based on the need of a periodic $\mathrm{HbAlc}$ measurement every 3 months, as well as reaching an equilibrium between reimbursement from health insurance companies and the burden on the caregiver teams. The lockdown secondary to the coronavirus disease (COVID-19) enabled assessing the significance of medical team input in a single visit.

The COVID-19 pandemic compelled the health public authorities to impose extreme steps to slow spread of the virus. Lockdown has been recognized as an effective measure for isolation and social distancing. On March 15, 2020, the Israeli population went under nationwide lockdown and medical access was limited. Thus, individuals with T1D were forced to miss their scheduled in-clinic visits.

Telehealth uses a technology-based virtual platform to deliver various aspects of health information, monitoring and medical care, including multidisciplinary team input [3,4]. Telehealth's largest segment is telemedicine, which is generally defined as the practice of medicine, by a physician, via a remote electronic interface. Telehealth involves multidisciplinary caregivers, including physicians, nurses, dietitians, psychosocial professionals, and educators [4].

The effectiveness of distal technologies in improving T1D management was previously reviewed [5]. The types of reported treatments included phone support, e-mailed medical support, behavioural therapy, text messaging, phone applications, and computer games. Most studies reported HbA1c as a primary outcome of improvement and examined long-term effects $[6,7]$. Additional outcome measures were diabetes knowledge, quality of life, and diabetes-related distress. Telehealth appears as an essential tool during the COVID-19 period [8], safe and effective for new-onset T1D [9]. However, we did not find reports on short-term effects of telehealth, in children with T1D.

Telehealth has not been common practice for T1D in real life in Israel. However, during the lockdown, our teams implemented facets of this modality. We used both real-time virtual consultation (synchronous) and stepwise consultation, in which data were uploaded for review by a clinician prior to a consult (asynchronous). The context was the delivery of medical, dietary, and behavioural care to children and youth with T1D. We assessed the feasibility of conducting a single telehealth multidisciplinary intervention, and its short-term impact on glycaemic metrics of young individuals with T1D. We hypothesized that telehealth visits during the COVID-19 lockdown would lead to short-term improvement in glycaemic control.

\section{Research design and methods}

\section{Research design}

This observational real-life multicentre study is based on data retrieved from medical files, continuous glucose monitoring systems (CGM), and continuous subcutaneous insulin infusion sets (pumps), acquired from Dexcom Clarity, CareLink, and Tidepool softwares. CGM and pump data were retrieved for the two weeks preceding and the two weeks after the meeting. All the meetings were conducted during the Israeli lockdown (from March 15, 2020, to April 12, 2020) by the paediatric diabetes multidisciplinary teams from the AWeSoMe study group.[10-12] The study group includes four paediatric diabetes multidisciplinary, university-affiliated clinics in Israel: Dana-Dwek Children's Hospital, Shamir Medical Center, Edmond and Lily Safra Children's Hospital and the National Juvenile Diabetes Center, Maccabi Health Care Services. The study was performed in accordance with the guidelines of the 2013 Declaration of Helsinki on human experimentation. Data confidentiality and patient anonymity were maintained at all times. The patients are not identifiable on an individual level either in this article or in the database. Due to the anonymous nature and mandatory collection of the information included in the dataset, informed consent from patients was not necessary. The study was approved by the local ethics committee of each medical centre.

\section{Study set-up and population}

Telehealth visits included video, telephone, and e-mail modalities and involved all the diabetes team members (physicians, nurses, dieticians, social workers, and psychologists), according to clinical care needs. The study population comprised all the individuals with T1D, aged $<25$ years, who were scheduled to have an in-clinic visit at the time of the COVID-19 lockdown, and instead had a telehealth visit. Technical support in downloading data from pumps and CGM devices was provided when needed. Of 195 individuals and families approached, 121 (62.0\%) downloaded complete data, with more than 70\% pump and CGM use (full data group). Of the $74(38.0 \%)$ with less usage or with incomplete data (partial data group), 36 (18.5\%) transferred partial data with handwritten doses of insulin and glucose metre readings, 20 (10.3\%) had technical difficulties downloading data, and 18 (9.2\%) requested to postpone the visit (Fig. 1).

\section{Data}

The information retrieved from medical files included: demographic characteristics (age, sex, households (single/ two parents), socio-economic position (SEP), by home 
Fig. 1 Flowchart of individuals with T1D who were scheduled to have an in-clinic visit at the time of the COVID-19 lockdown. The diagram shows the numbers of patients who did and did not have telehealth visits, according to their having full downloaded data available. CGM: continuous glucose monitor

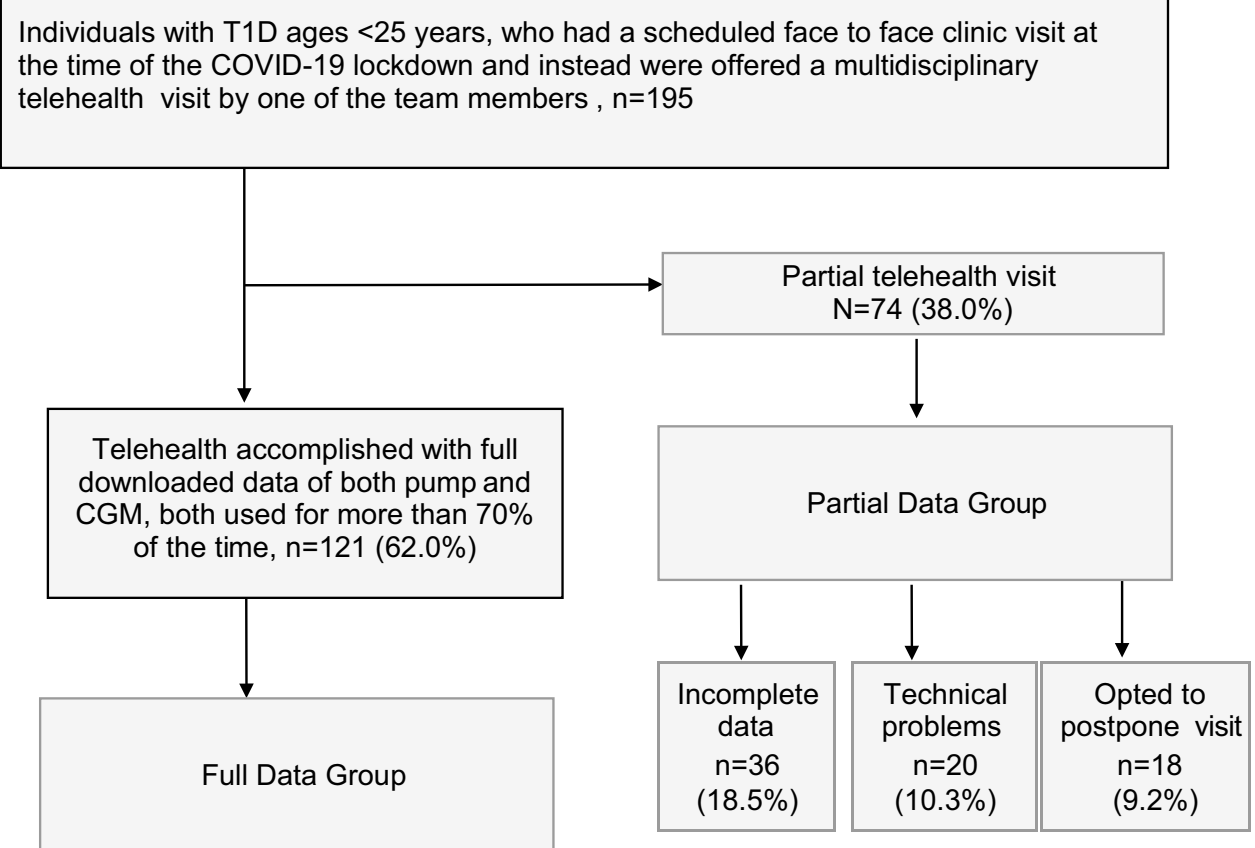

address (SEP cluster and SEP index), based on the Israel Central Bureau of Statistics' Socio-Economic Level of the Population 2015 [13], diabetes duration, mode of insulin therapy (multiple daily injections or pump) and coexistent morbidity including autoimmune diseases (thyroid and celiac) and attention-deficit hyperactivity disorder.

The information retrieved from the two-week ambulatory glucose profile reports included: percent time spent in various glycaemic ranges: time-in-range (TIR) (70-180mg/dL; 3.9-10 mmol/L), severe hypoglycaemic range $(<54 \mathrm{mg} / \mathrm{dL} ;<3 \mathrm{mmol} / \mathrm{L})$, mild hypoglycaemic range $(54-70 \mathrm{mg} / \mathrm{dL} ; 3-3.8 \mathrm{mmol} / \mathrm{L})$, moderate hyperglycaemic range (180-250 mg/dL; $10-13.3 \mathrm{mmol} / \mathrm{L})$, and severe hyperglycaemia $(>250 \mathrm{mg} / \mathrm{dL} ;>13.3 \mathrm{mmol} / \mathrm{L})$; coefficient of variation (CV), percent time CGM active, mean glucose levels (mg/dL), and estimated HbA1c (\%, $\mathrm{mmol} / \mathrm{l}$ ) glucose management indicator (GMI) [14].

The information retrieved from the two-week pump downloaded reports included the reported amount of carbohydrates, the mean total daily dose of insulin (TDDi), and the mean percent of basal or long-acting insulin.

The relative change in TIR was calculated as the ratio of the difference in TIR for the two-week period after, compared to the two-week period prior, the telehealth visit (TIR after-TIR before), and the patient's potential improvement (100-TIR before)*100. This parameter enables distinguishing those with the most room for improvement (lower initial TIR) from those who were already mostly in the target TIR, and had little room to improve.
Behavioural characteristics of the lockdown period were assessed, including sleeping pattern, participation in school zooming and physical activity. At the end of the telehealth visit, individuals with T1D and their family members were asked whether they had benefited from the telehealth visit and whether they were interested in future telehealth visits in addition to in-clinic visits. The possible response ranged from 1 to 5 on a Likert scale, from not beneficial and not interested, to very beneficial and very interested.

\section{Outcome measures}

Primary outcome measures were: (a) the relative change in TIR after the telehealth visit, and (b) the parameters associated with relative change in TIR. The secondary outcome measures were: (a) the characteristics of patients for whom a telehealth visit may not be suitable.

\section{Statistical analysis}

Power analysis showed that a sample size of 113 would achieve $80 \%$ power to detect a difference of $4.0 \%$ in TIR between the actual mean of 7.0 and the null-hypothesized mean of 3.0, with an estimated standard deviation of 15.0 and with a significance level (alpha) of 0.050 using a twosided one-sample t-test.

Categorical variables were described using frequencies and percentages. Continuous variables were examined for normal distribution using histograms, Q-Q plots, and 
Kolmogorov-Smirnov test. Normally distributed continuous variables were expressed as means \pm standard deviations (SDs) and skewed variables as medians and interquartile ranges. The cohort was stratified according to full and partial availability of downloaded data for telehealth visits. Groups were compared using the Chi-Square test or Fisher's exact test for categorical variables and independent samples t-test or the Mann-Whitney test for ordered and continuous variables, as appropriate. Paired sample t-test and Wilcoxon test were used to compare continuous variables between the two 2-week periods examined. The McNemar test was used to compare categorical variables, Pearson correlation coefficient to evaluate associations between normally distributed continuous variables, and Spearman correlation coefficient to evaluate associations between continuous variables not normally distributed. Associations between relative improvement in TIR and categorical variables were examined using independent sample t-test or ANOVA. Multivariate linear regression was used to examine the association between relative improvement in TIR and all automated metrics derived from pumps and CGMS, prior to the intervention. The backward method was applied for variate selection. $P>0.1$ was a criterion for removal. The linear regression was evaluated to meet assumptions (lack of multicollinearity-VIF $<5$, normal distribution and homoscedasticity of the residuals). All statistical tests were two-sided. Statistical significance was defined as $p<0.05$. The statistical analyses were performed by SPSS software (IBM SPSS STATISTIC version 24, IBM CORP., Armonk, NY, USA, 2016).

\section{Results}

The study cohort comprised 195 individuals (47.7\% males), mean \pm SD age $14.6 \pm 5.3$ years (range 1.0-24.7 years), mean age at T1D diagnosis $8.6 \pm 4.3$ years, and mean diabetes duration $6.0 \pm 4.6$ years. The demographic, socio-economic, and clinical characteristics of the study population, according to data availability (full data and partial data groups), are presented in Table 1. Individuals with full data were younger $(13.4 \pm 5.3$ vs. $16.5 \pm 4.4, p<0.001)$, had higher SEP index and cluster, had shorter diabetes duration $(5.2 \pm 4.4$ vs. 7.1 $\pm 4.7, p<0.001$ ), and used pumps ( $80 \%$ vs. $63 \%, p<0.001)$.

\section{Associations of telehealth with glycaemic control parameters}

Table 2 presents CGM metrics, pump data, and behavioural parameters prior and two-week post-telehealth visits for 121 individuals with full downloaded data.

Glycaemic ranges improved significantly following the telehealth visits. The percent of TIR increased from

Table 1 Sociodemographic and diabetes-related characteristics of paediatric patients with type 1 diabetes (T1D) stratified by the availability of full or partial downloaded data during telehealth intervention

\begin{tabular}{|c|c|c|c|c|}
\hline & & Full data group $N=74$ & Partial data group $N=121$ & $p$ value \\
\hline \multirow[t]{6}{*}{ Demographic characteristics } & Sex, male $(\%)$ & 51.4 & 45.5 & 0.424 \\
\hline & Age, years & $16.5 \pm 4.4$ & $13.4 \pm 5.3$ & $<0.001$ \\
\hline & SEP cluster & $7.0(5,8)$ & $8(7,9)$ & $<0.001$ \\
\hline & SEP index & $0.5(0.1,1.3)$ & $1.2(0.5,1.8)$ & $<0.001$ \\
\hline & Two-parent household (\%) & 78.1 & 86.8 & 0.11 \\
\hline & Number of children & $3 \pm 1$ & $3 \pm 1$ & 0.23 \\
\hline \multirow[t]{5}{*}{ Diabetes-related characteristics } & Age at T1D diagnosis, years & $9.4 \pm 4.5$ & $8.1 \pm 4.1$ & 0.05 \\
\hline & Diabetes duration, years & $7.1 \pm 4.7$ & $5.3 \pm 4.4$ & 0.006 \\
\hline & $* \mathrm{HbA} 1 \mathrm{c}, \%$ & $7.9(7.0,8.5)$ & $7.6(6.9,8.1)$ & 0.081 \\
\hline & $* \mathrm{HbA} 1 \mathrm{c}, \mathrm{mmol} / \mathrm{mol}$ & $63.0(53.0,69.0)$ & $60.0(52.0,65.0)$ & \\
\hline & Insulin pump therapy & 63.5 & 80.2 & $<0.001$ \\
\hline \multirow[t]{3}{*}{ Comorbid conditions (\%) } & Celiac disease & 8.1 & 13.2 & 0.27 \\
\hline & Hashimoto thyroiditis & 6.8 & 4.1 & 0.51 \\
\hline & ADHD & 16.2 & 24.0 & 0.48 \\
\hline \multirow[t]{3}{*}{ Means of telemedicine } & Video call & 33.8 & 85.1 & $<0.001$ \\
\hline & Telephone call & 62.2 & 12.4 & \\
\hline & E-mail mean of contact & 4.1 & 2.5 & \\
\hline
\end{tabular}

Data are presented as mean \pm standard deviation for normally distributed parameters, median and interquartile range for skewed parameters, and percent for categorical data

*HbA1c drawn at the last clinic visit

ADHD, Attention-deficit/hyperactivity disorder; CGM, Continuous Glucose Monitoring, SEP, socio-economic position, T1D, type 1 diabetes 
$59.0 \pm 17.2$ to $62.9 \pm 16.0 \%, p<0.001$. The percent time spent in the severe hypoglycaemic range decreased significantly: from 0.5 (IQR 0,2 ) to 0.5 (IQR $0,1.1$ ), as did the percent time in the hyperglycaemic range: from $32.0 \%$ (IQR 20.0, 42.1) to $28.6 \%$ (IQR 19.0, 37.3), to $p=0.005$. The respective mean glucose levels were $164 \pm 29 \mathrm{mg} / \mathrm{dL}$ and $160 \pm 26 \mathrm{mg} / \mathrm{dL}, p=0.001$; and the estimated $\mathrm{HbA} 1 \mathrm{c}$ (GMI): $56.8 \pm 9.3 \mathrm{mmol} / \mathrm{mol}(7.35 \pm 0.85 \%)$ and $55.2 \pm$ $8.9 \mathrm{mmol} / \mathrm{mol}(7.20 \pm 0.81 \%), p<0.001$

The mean change in relative-TIR was $5.7 \pm 26.1 \%$. Table 3 presents parameters associated with improvement in relative-TIR in a univariate analysis. These parameters included living in a single-parent household, and a number of initial glycaemic parameters: higher mean glucose level, less time spent at range, and at hypoglycaemic range $<54 \mathrm{mg} / \mathrm{dL}$, more time spent in the $180-250 \mathrm{mg} / \mathrm{dL}$ range, higher TDDi, and a greater amount of carbohydrate consumption reported. A multiple regression logistic analysis that included all automated metrics derived from pump and CGMS parameters demonstrated only initial lower TIR (OR -0.506 [95\%CI $-0.99,-0.023], p=0.04$ ) and living in a single-parent household (OR 13.8 [95\% CI $0.6,27.1], p=0.04$ ) (Fig. 2) to be significant. These two characteristics explained $18 \%$ of the improvement in relative-TIR.

\section{Patient satisfaction from telehealth}

All patients and parents from the full-data group answered the questionnaires. Of them, $86.7 \%$ reported significant benefit and $74.2 \%$ were very interested in combining telehealth in future management. Of 74 individuals from the partial data group, 34 (45.9\%) had telehealth visits, 22 (64.7\%) reported considerable benefit and $18(53.6 \%)$ were very interested in combining telehealth in future management. These differences were significant between the groups, $p=0.047$ for assessment of benefit, and $p=0.008$ for future interest in telehealth.

\section{Discussion}

This real-life study demonstrated the short-term effectiveness of a synchronous/asynchronous telehealth visit, performed during the COVID-19 pandemic as a substitute for the traditional scheduled in-clinic visit. A telehealth visit with full available glycaemic parameters was achieved in two-thirds of our cohort. During the two weeks after the telehealth visit, significant improvement was shown in TIR and GMI, with less time spent in the severe hypoglycaemic and hyperglycaemic ranges. Individuals who benefitted most from telehealth visits were those with lower baseline TIR and from single-parent households.
Table 2 Glucose metrics, insulin requirements, and behaviour characteristics for the two weeks preceding and the two weeks following telehealth visits, for individuals from the full data group, $n=121$

\begin{tabular}{lllc}
\hline & Before telehealth visit & After telehealth visit & $P$ value \\
\hline \% Time-in-range, $(70-180 \mathrm{mg} / \mathrm{dl})$ & $59.0 \pm 17.2$ & $62.9 \pm 16.0$ & $<0.001$ \\
\% Time $<54 \mathrm{mg} / \mathrm{dL}$ & $0.5(0.0,2)$ & $0.5(0.0,1.1)$ & 0.05 \\
\% Time in 54-70 mg/dL & $2.6(1.0,5.3)$ & $2.3(1.0,5.0)$ & 0.87 \\
\% Time in 180-250 mg/dL & $32.0(20.0,42.1)$ & $28.6(19.0,37.3)$ & 0.005 \\
\% Time $>$ 250 mg/dL & $8.8(3.6,19.0)$ & $8.0(2.0,12.6)$ & $<0.001$ \\
Coefficient of variation, \% & $37.1 \pm 7.1$ & $35.6 \pm 7.2$ & 0.002 \\
\% Time CGM Active & $92.8(85.0,97.2)$ & $92.4(85.8,97.1)$ & 0.16 \\
Mean glucose, mg/dL & $164 \pm 29$ & $160 \pm 26$ & 0.001 \\
Estimated HbA1c (GMI), \% & $7.35 \pm 0.85$ & $7.20 \pm 0.81$ & $<0.001$ \\
Estimated HbA1c (GMI), mmol/mol & $56.8 \pm 9.3$ & $55.2 \pm 8.9$ & \\
Mean TDD (unit/kg/d) & $0.8(0.6,1.9)$ & $0.8(0.6,1.0)$ & 0.19 \\
\% Basal insulin per day & $44(38,54)$ & $45(39,51.5)$ & 0.55 \\
Mean daily carbohydrate (gram) & $135(100,175)$ & $140(108,178)$ & 0.48 \\
Physical activity (hours/week) & $2(0,4)$ & $3(1,5)$ & $<0.001$ \\
\#Sleep pattern changes $\%$ of patients) & 31.9 & 29.2 & 0.47 \\
*Scholl zooming (\% of patients) & 75.7 & 86.8 & 0.008 \\
\hline
\end{tabular}

Data are presented as mean \pm standard deviation for normally distributed numerical data, median and interquartile range for skewed numerical data, and percent for categorical data

*School zooming was applicable for 6-16-year-old patients only, $n=74$.

\#Sleep pattern changes were applicable for 6-25-year-old patients only, $n=116$

CGM, continuous glucose monitoring, TDD-total daily dose, GMI, glycaemic metabolic index 
Table 3 Correlations between improvement in relativetime in range (TIR) and sociodemographic, behavioural, and diabetes-related characteristics of the study cohort, $n=118$

\begin{tabular}{|c|c|c|c|}
\hline \multirow[t]{2}{*}{ Characteristic } & & \multicolumn{2}{|c|}{ Relative change in TIR } \\
\hline & & $\begin{array}{l}\text { r-Correlation } \\
\text { coefficient }\end{array}$ & $P$ value \\
\hline \multirow[t]{2}{*}{ Demographics } & Sex & NA & 0.69 \\
\hline & Age & -0.02 & 0.86 \\
\hline \multirow[t]{14}{*}{ Diabetes-related characteristics } & Age at T1D diagnosis & -0.04 & 0.64 \\
\hline & Diabetes duration & -0.04 & 0.70 \\
\hline & Prior mean glucose & 0.22 & 0.015 \\
\hline & Prior $* \mathrm{HbA} 1 \mathrm{c}$ & -0.05 & 0.60 \\
\hline & Prior time-in-range & -0.35 & $<0.001$ \\
\hline & Prior time $<54 \mathrm{mg} / \mathrm{dL}$ & -0.20 & 0.04 \\
\hline & Prior time $54-70 \mathrm{mg} / \mathrm{dL}$ & -0.10 & 0.29 \\
\hline & Prior time $180-250 \mathrm{mg} / \mathrm{dL}$ & 0.31 & 0.001 \\
\hline & Prior time $>250 \mathrm{mg} / \mathrm{dL}$ & 0.17 & 0.08 \\
\hline & Prior coefficient of variation & -0.03 & 0.75 \\
\hline & Prior $\%$ time CGM active & 0.00 & 0.97 \\
\hline & Prior TDD (units/kg/d) & 0.28 & 0.003 \\
\hline & Prior basal & -0.18 & 0.06 \\
\hline & Prior mean daily carbohydrates (gram/d) & 0.34 & 0.001 \\
\hline \multirow[t]{3}{*}{ Behavioural } & ${ }^{\#}$ Sleep pattern & NA & 0.36 \\
\hline & Physical activity & 0.000 & 0.99 \\
\hline & ${ }^{\$}$ School zooming & NA & 0.26 \\
\hline \multirow[t]{3}{*}{ Medical team recommendations } & Dietary recommendations & NA & 0.09 \\
\hline & Insulin recommendations & NA & 0.23 \\
\hline & Behavioural recommendations & NA & 0.95 \\
\hline \multirow[t]{4}{*}{ Social data } & SEP cluster & -0.05 & 0.60 \\
\hline & SEP index & -0.05 & 0.58 \\
\hline & One-parent household & NA & 0.003 \\
\hline & Number of children & -0.11 & 0.24 \\
\hline \multirow[t]{3}{*}{ Medical state } & Celiac disease & NA & 0.95 \\
\hline & Hashimoto thyroiditis & NA & 0.27 \\
\hline & ADHD & NA & 0.78 \\
\hline
\end{tabular}

$\mathrm{r}$ - Pearson correlation coefficient and Spearman's correlation coefficient were used to evaluate associations between normally and abnormally distributed continuous variables, accordingly.

NA -The association between the relative improvement in TIR and categorical variables was studied using independent sample T-test or ANOVA.

${ }^{\wedge}$ For three of the 121 participants with full CGM data, TIR could not be assessed due to technical reasons.

${ }^{*} \mathrm{HbA} 1 \mathrm{c}$ drawn at the last clinic visit, during the last 6 months from the digital visit.

${ }^{\$}$ School zooming were applicable for ages 6-16 years only, $n=74$.

"Sleep pattern changes were applicable for ages 6-25 years only, $n=116$

CGM, continuous glucose monitoring, TDD, total daily dose, ADHD, attention-deficit/hyperactivity disorder, SEP, socio-economic position.
During the COVID-19 period, telehealth need was acute, and its effect in prevention of DKA [15] and in management in new-onset T1DM were reported [9]. Furthermore, we showed that the lockdown itself, without intervention had no detrimental effect on glycaemic control [16]. The first lesson from this study is that interventions and diabetes plans should be adapted according to circumstances. This lesson is not relevant only to the COVID-19 pandemic lockdown, but should be extrapolated to health care in normal periods. For those enthusiastic about the approach, periodic telehealth visits could help maintain the frequency of follow-up in the non-COVID19 era, by mitigating for long distances from paediatric diabetes centres. One study reported a high rate of missed visits, especially among patients with poor glycaemic control, and concluded that approaches to maximizing attendance, such as virtual visits should be explored [17]. 
Fig. 2 Multiple regression logistic analysis. A multiple regression logistic analysis of parameters that were associated with individual improved relative-TIR

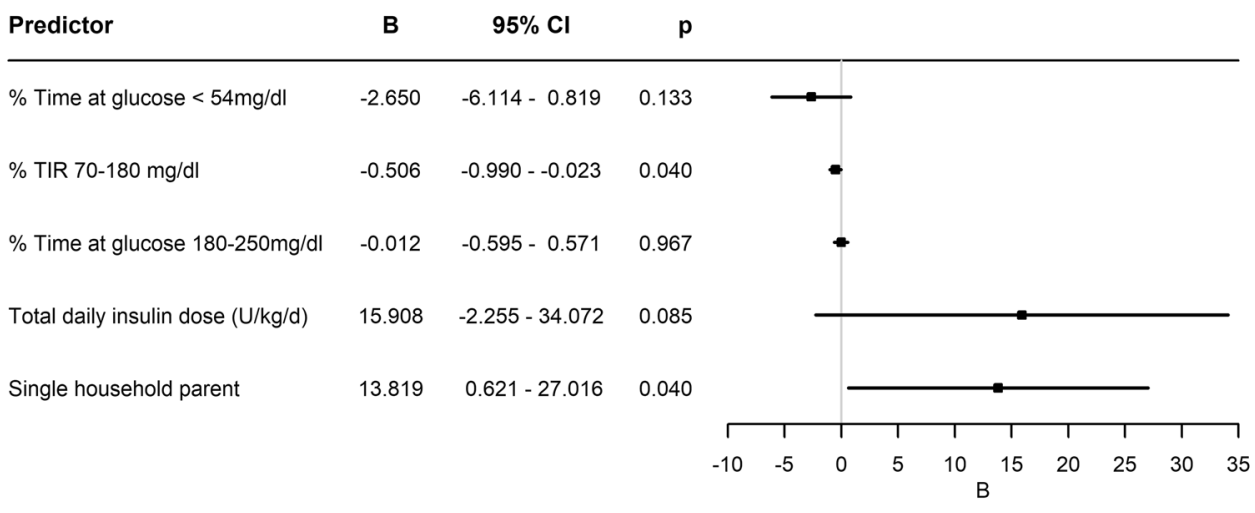

Furthermore, frequently cancelled appointments are associated with frustration with self-care [18] and with deterioration in glycaemic control $[17,19]$. Therefore, efforts should be made not to postpone or miss visits, and clinicians should reinforce families for keeping close contact with their medical teams. In real life, the immediate impact of in-clinic visits and changes in insulin plans made by the medical team are hard to evaluate. Between visits, various life circumstances may impact glucose level such as weight gain, holidays, vacations, and participation in sport activities. The COVID-19 lockdown enabled us to assess the benefit of a single telehealth contact with the medical team. It is reassuring that CGM metrics improved despite the challenging situation of lockdown.

Percentages of TIR and of time spent in the severe hypoglycaemic and hyperglycaemic ranges may be the preferred metric for determining outcomes of clinical studies that assess glycaemic control [20]. This study demonstrates improvement in glycaemic control, by significantly less time spent in the severe hypoglycaemic range and less time spent in both hyperglycaemic and severe hyperglycaemic ranges, and more time spent in range. Treatment of severe hypoglycaemic episodes is often associated with elevated glucose levels post event. Furthermore, the unpleasant symptoms and negative consequences associated with hypoglycaemia may result in significant anxiety or even a fear of hypoglycaemia, which is one of the chief barriers to optimal glycaemic control in T1D [21]. Therefore, successful reductions in time spent in the severe hypoglycaemic and in the hyperglycaemic ranges possibly reflect the importance of close contact with the medical team.

The second lesson learned from this study is that telehealth visits are beneficial to diverse populations. Previous studies assessing telemedicine in individuals with T1D have shown the mixed results. A meta-analysis reported a role of telemedicine in glycaemic management, particularly in adults [22]. However, the studies examined were characterized by a high level of heterogeneity, including diversity of definitions of telemedicine. These included devices such as telephone, modems, mobile phones, and websites; thus, assessing treatment efficacy was difficult [22]. Moreover, most studies assessed the long-term impact of telehealth [4]. We assessed the short-term benefit of a single telehealth visit, according to downloaded data on glycaemic control. We assessed the change in TIR after intervention per individual. The clinical relevance of the effect of a change in TIR depends on the initial TIR. Individuals with lower TIR need a greater change for the improvement to be clinically meaningful. Therefore, we used the relative-TIR parameter to analyse characteristics of individual patients who improved significantly. Our findings show that those who most improved from the telehealth visit had lower TIR prior the telehealth visit. This concurs with previous interventions that showed greater benefit to patients with suboptimal or poor glycaemic control than to those with good control [23]. This finding suggests that tailoring telehealth visits in between in-clinic visits may improve adherence and glycaemic control, especially among those needing it the most.

The complexity of T1D treatment requires intensive involvement of families in the diabetes care of their children. Data from two decades ago show that children with diabetes from single-mother families had poorer glycaemic control than children from two-parent families [24]. Age and missed clinic appointments predicted $\mathrm{HbA} 1 \mathrm{c}$ levels in those single-mother families [24]. In the current study, lone parenting versus two-parent households was associated with greater improvement in relative-TIR. We speculate that staying at home during the COVID-19 lockdown enabled single parents to be more involved in the treatment and to improve glucose control.

For $18 \%$ of the individuals with T1D with a scheduled in-clinic visit during the COVID-19 lockdown, glycaemic data could not be downloaded and were only available by fax or e-mail. This precluded calculating glycaemic parameters. Nevertheless, $65 \%$ of these patients and their parents were satisfied with the telehealth visit. This compares with $90 \%$ of those for whom full glycaemic data were downloaded. Furthermore, $50 \%$ and $75 \%$, respectively, were very interested in combining telehealth in future management with in-clinic visits. Previous studies showed that the 
opportunity to connect remotely to a caregiver promotes patient satisfaction and engagement [25, 26]. Nine percent of the families in our study opted to postpone telehealth visits. This collaborates reports that some families were not willing to replace in-clinic visits with telehealth visits, had technical problems, or lacked the skills needed to participate in a telehealth visit. Therefore, telehealth appears not to be a solution for all individuals. Personal tailoring of treatment is needed.

This study has some limitations. We did not examine the effectiveness of the telehealth visit in normal life of changing physical activity, eating out, and concerns relating to schooling and interacting with peers. The benefits of face-to-face and telehealth visits were not compared; therefore, we can only suggest telehealth as a tool when distance or clinic visit are an issue. Further, we did not compare our findings to individuals who did not have any visit during the COVID-19 lockdown, as previously described to be of no worsening effect [16, 27, 28].

In conclusion, although the setting of our study during the COVID-19 lockdown was a unique period of time, our findings highlight the benefit of close encounters with the medical staff, specifically for those with poorer glycaemic control. We believe that in-clinic, face-to-face visits are essential, particularly for children, who need physical examinations and evaluations of their growth and puberty. However, since frequent adjustments in insulin doses are integral to T1D management, add-on telehealth visits in between in-clinic visits in a suitable setting for children and their families may be beneficial and should be part of our tool-box for management.

Acknowledgement We would like to thank Dr. Ziv Tomer from the Department of Epidemiology and Preventive Medicine, School of Public Health, Tel Aviv University, for the statistical analysis.

Author contributions MR and OPH made substantial contributions to the conception and design of the study, acquisition, analysis, and interpretation of data and drafting the initial manuscript and revising it critically for important intellectual content. KM-A, NL, GB, NB, MD, and EC contributed to the data used in this study, contributed to the discussion and reviewed and edited the manuscript. ZL and YL made substantial contributions to the conception and design of the study, the interpretation of data, contributed to the discussion, reviewed and edited the manuscript for important intellectual content. All authors gave final approval of the version to be published. OPH is the guarantor of this work and, as such, had full access to all the data in the study and takes responsibility for the integrity of the data and the accuracy of the data analysis.

Funding Not applicable

Data availability The datasets generated during and/or analysed during the current study are available from the corresponding author on reasonable request.

\section{Compliance with ethical standards}

Conflict of interest The authors declare that they have no conflict of interest

\section{References}

1. American Diabetes A (2020) Children and adolescents: standards of medical care in diabetes-2020. Diabet Care 43:S163-S82. https ://doi.org/10.2337/dc20-S013

2. Pihoker C, Forsander G, Fantahun B, Virmani A, Corathers S, Benitez-Aguirre $\mathrm{P}$ et al (2018) ISPAD Clinical practice consensus guidelines 2018: the delivery of ambulatory diabetes care to children and adolescents with diabetes. Pediatr Diabet 19(Suppl 27):84-104. https://doi.org/10.1111/pedi.12757

3. Tuckson RV, Edmunds M, Hodgkins ML (2017) Telehealth. New Engl J Med 377(16):1585-1592

4. Duke DC, Barry S, Wagner DV, Speight J, Choudhary P, Harris MA (2018) Distal technologies and type 1 diabetes management. Lancet Diabet Endocrinol 6(2):143-56. https://doi.org/10.1016/ S2213-8587(17)30260-7

5. Shan R, Sarkar S, Martin SS (2019) Digital health technology and mobile devices for the management of diabetes mellitus: state of the art. Diabetologia. 62(6):877-87. https://doi.org/10.1007/s0012 5-019-4864-7

6. Yaron M, Sher B, Sorek D, Shomer M, Levek N, Schiller T et al (2019) A randomized controlled trial comparing a telemedicine therapeutic intervention with routine care in adults with type 1 diabetes mellitus treated by insulin pumps. Acta Diabetol 56(6):667-73. https://doi.org/10.1007/s00592-019-01300-1

7. Bertuzzi F, Stefani I, Rivolta B, Pintaudi B, Meneghini E, Luzi L et al (2018) Teleconsultation in type 1 diabetes mellitus (TELEDIABE). Acta Diabetol 55(2):185-92. https://doi.org/10.1007/ s00592-017-1084-9

8. Jones MS, Goley AL, Alexander BE, Keller SB, Caldwell MM, Buse JB (2020) Inpatient transition to virtual care during COVID19 pandemic. Diabet Technol Ther 22(6):444-8. https://doi. org/10.1089/dia.2020.0206

9. Garg SK, Rodbard D, Hirsch IB, Forlenza GP (2020) Managing new-onset type 1 diabetes during the COVID-19 pandemic: challenges and opportunities. Diabet Technol Ther 22(6):431-9. https ://doi.org/10.1089/dia.2020.0161

10. Rachmiel M, Levy-Shraga Y, Gruber N, Pinhas-Hamiel O, Barash G, Pivko-Levy D et al (2019) Comparing insulin pump devices in real life: the AWeSoMe study group prospective experience. Diabet Technol Ther 21(3):138-45. https://doi.org/10.1089/ dia.2018.0309

11. Landau Z, Abiri S, Gruber N, Levy-Shraga Y, Brener A, Lebenthal $\mathrm{Y}$ et al (2018) Use of flash glucose-sensing technology (FreeStyle Libre) in youth with type 1 diabetes: AWeSoMe study group real-life observational experience. Acta Diabetol 55(12):1303-10. https://doi.org/10.1007/s00592-018-1218-8

12. Rachmiel M, Landau Z, Boaz M, Mazor Aronovitch K, Loewenthal N, Ben-Ami M et al (2015) The use of continuous glucose monitoring systems in a pediatric population with type 1 diabetes mellitus in real-life settings: the AWeSoMe study group experience. Acta Diabetol 52(2):323-9. https://doi.org/10.1007/s0059 2-014-0643-6

13. Israel Central Bureau of Statistics (CBS) (2019) Characterization and Classification of Geographical Units by the Socio-Economic Level of the Population, 2015. https://www.cbs.gov.il/he/publi cations/DocLib/2019/1765_socio_economic_2015/e_print.pdf 
14. Bergenstal RM, Beck RW, Close KL, Grunberger G, Sacks DB, Kowalski A et al (2018) Glucose management indicator (GMI): a new term for estimating A1C from continuous glucose monitoring. Diabet Care 41(11):2275-80. https://doi.org/10.2337/ dc18-1581

15. Peters AL, Garg SK (2020) The silver lining to COVID-19: avoiding diabetic ketoacidosis admissions with telehealth. Diabet Technol Ther 22(6):449-53. https://doi.org/10.1089/dia.2020.0187

16. Brener A, Mazor-Aronovitch K, Rachmiel M, Levek N, Barash G, Pinhas-Hamiel O et al (2020) Lessons learned from the continuous glucose monitoring metrics in pediatric patients with type 1 diabetes under COVID-19 lockdown. Acta Diabetol 57(12):15117. https://doi.org/10.1007/s00592-020-01596-4

17. Markowitz JT, Volkening LK, Laffel LM (2014) Care utilization in a pediatric diabetes clinic: cancellations, parental attendance, and mental health appointments. J Pediatr 164(6):1384-9. https ://doi.org/10.1016/j.jpeds.2014.01.045

18. Weinger K, McMurrich SJ, Yi JP, Lin S, Rodriguez M (2005) Psychological characteristics of frequent short-notice cancellers of diabetes medical and education appointments. Diabet Care 28(7):1791-3. https://doi.org/10.2337/diacare.28.7.1791

19. Kofoed PE, Thomsen J, Ammentorp J (2010) An unplanned delay between control visits influences the metabolic status in children with diabetes: an observational study. Acta Paediatr 99(5):774-7. https://doi.org/10.1111/j.1651-2227.2009.01673.x

20. Wright LA, Hirsch IB (2017) Metrics beyond hemoglobin A1C in diabetes management: time in range, hypoglycemia, and other parameters. Diabet Technol Ther 19(S2):S16-S26. https://doi. org/10.1089/dia.2017.0029

21. Realsen JM, Chase HP (2011) Recent advances in the prevention of hypoglycemia in type 1 diabetes. Diabet Technol Ther 13(12):1177-86. https://doi.org/10.1089/dia.2011.0150

22. Lee SWH, Ooi L, Lai YK (2017) Telemedicine for the management of glycemic control and clinical outcomes of type 1 diabetes mellitus: a systematic review and meta-analysis of randomized controlled studies. Front Pharmacol. https://doi.org/10.3389/fphar .2017 .00330

23. Pillay J, Armstrong MJ, Butalia S, Donovan LE, Sigal RJ, Vandermeer B et al (2015) Behavioral programs for type 2 diabetes mellitus: a systematic review and network meta-analysis. Ann Intern Med 163(11):848-60. https://doi.org/10.7326/m15-1400

24. Thompson SJ, Auslander WF, White NH (2001) Comparison of single-mother and two-parent families on metabolic control of children with diabetes. Diabet Care 24(2):234-8. https://doi. org/10.2337/diacare.24.2.234

25. Orlando JF, Beard M, Kumar S (2019) Systematic review of patient and caregivers' satisfaction with telehealth videoconferencing as a mode of service delivery in managing patients' health. PLoS One. https://doi.org/10.1371/journal.pone.0221848

26. Raymond JK, Berget CL, Driscoll KA, Ketchum K, Cain C, Fred Thomas JF (2016) CoYoT1 Clinic: innovative telemedicine care model for young adults with type 1 diabetes. Diabet Technol Ther 18(6):385-90. https://doi.org/10.1089/dia.2015.0425

27. Bonora BM, Boscari F, Avogaro A, Bruttomesso D, Fadini GP (2020) Glycaemic control among people with type 1 diabetes during lockdown for the SARS-CoV-2 outbreak in Italy. Diabet Ther. https://doi.org/10.1007/s13300-020-00829-7

28. Beato-Víbora PI (2020) No deleterious effect of lockdown due to COVID-19 pandemic on glycaemic control, measured by glucose monitoring, in adults with type 1 diabetes. Diabet Technol The. https://doi.org/10.1089/dia.2020.0184

Publisher's Note Springer Nature remains neutral with regard to jurisdictional claims in published maps and institutional affiliations. 